\title{
A Review on Different Configuration of Electrochemical Discharge Micro-machining Process
}

\author{
Shalini Sahu ${ }^{1}$ and Anshuman Srivastava ${ }^{2 *}$ \\ ${ }^{1} P G$ Student, Department of Mechanical Engineering, India \\ ${ }^{2}$ Professor, Department of Mechanical Engineering, India \\ *Corresponding author: Anshuman Srivastava, Professor, Department of Mechanical Engineering, India
}

Submission: 啡 July 17, 2018 ; Published: 眥 August 16, 2018

\begin{abstract}
The new developments in engineering materials such as titanium alloys, high performance steels, precious metals, ceramics, composites, polymers, high strength alloys, thin film nano-structured flexible electronics, photopolymers etc requires advanced machining process for its shaping and to be functional. Electrochemical discharge machining process is a high capability machining process and suitable for different conducting and nonconducting materials irrespective to their strength. This machining process has a combined effect of electrochemical machining process and electro discharge machining process both. A number of variants have been developed for making symmetrical and unsymmetrical holes, straight and curved channels, slitting, cutting etc by using phenomena of electrochemical machining process. Present paper deals with the different configurations of electrochemical discharge micro machining process.
\end{abstract}

Keywords: ECDM; Hybrid micro machining process; Configuration of ECSMM

\section{Introduction}

Advanced engineering materials have high potential for their applications in various fields of engineering due to their special properties such as high strength, high thermal resistance high wear resistance high hardness. The materials like titanium alloys, high performance steels, precious metals, faces economical challenge during its conventional machining. The composites and polymers materials faces problem of fiber pull out, material deformations, heat affected zone during its conventional machining. Electrochemical discharge machining (ECDM) can be successfully applied for machining of different conducting and non-conducting materials, hard and brittle materials, chemically active and nonactive materials etc.

Rajurkar et al. [1] defines hybrid machining process as a combination of two or more processes to remove material. These hybrid processes are developed to enhance advantages and to minimize potential disadvantages found in an individual technique. He also writes that the performance characteristics of hybrid micro machining processes must be considerably different from those that are characteristic for the component processes when performed separately. In hybrid machining processes, all constituent processes are directly involved in the material removal and in assisted micro machining process only one of the participating processes directly removes the material while the other only assists in removal by positively changing the conditions of machining. Following are some example of hybrid micro-machining processes. Figure 1 shows the configuration of hybrid micro machining processes and they are tabulated in Table 1; and show the some of the assisted and hybrid micro-machining processes.

Table 1: Table of assisted and hybrid micro machining processes.

\begin{tabular}{|c|c|}
\hline $\begin{array}{c}\text { Assisted Micromachining } \\
\text { Process }\end{array}$ & Hybrid Micromachining Process \\
\hline Vibration assisted turning & Grinding EDMM \\
\hline Vibration assisted milling & Grinding ECMM \\
\hline Vibration assisted drilling & Drilling ECSMM \\
\hline Vibration assisted polishing & Travelling wire ECSMM \\
\hline Vibration assisted grinding & Electrolytic in-process dressing \\
\hline Vibration assisted EDMM & Chemo-mechanical polishing \\
\hline LASER assisted turning & \\
\hline LASER assisted milling & \\
\hline LASER assisted jet ECMM & \\
\hline LASER drilling assisted by jet & \\
\hline ECMM & \\
\hline
\end{tabular}

Volume - 7 Issue - 4 


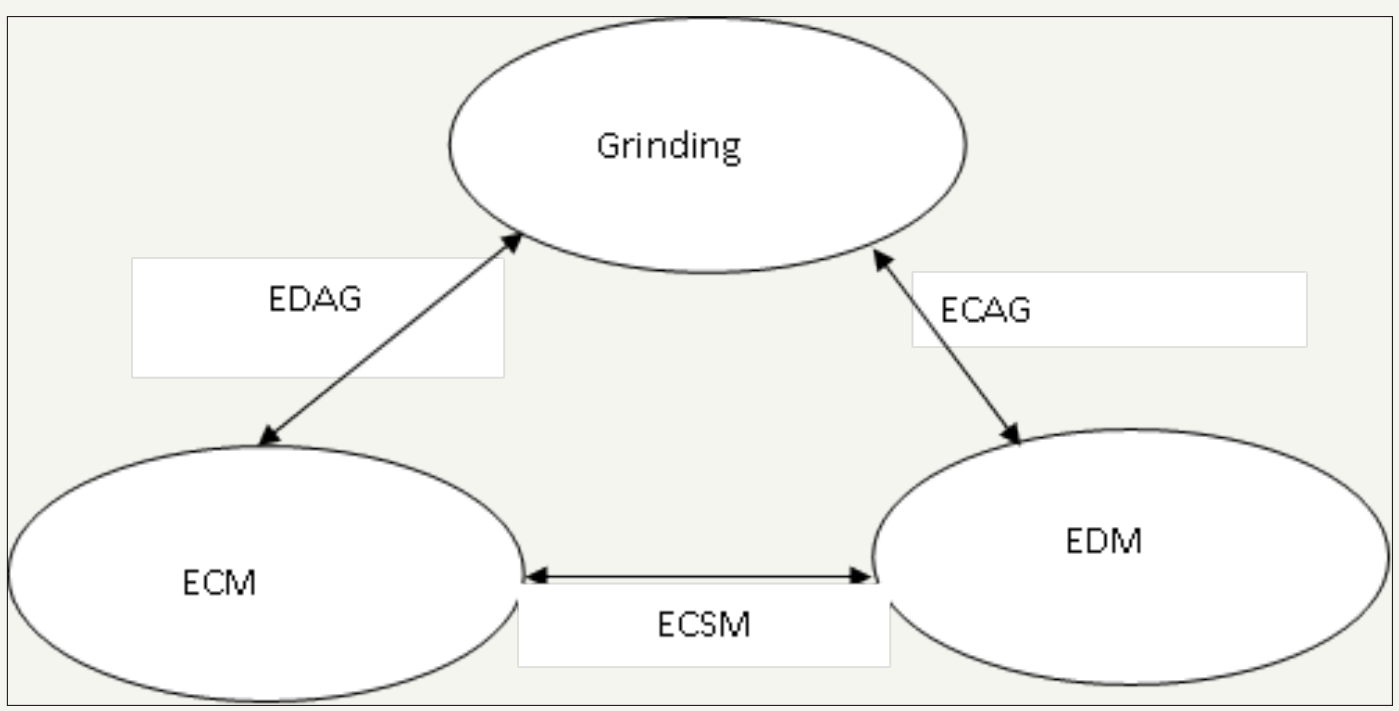

Figure 1: Configuration of hybrid machining system.

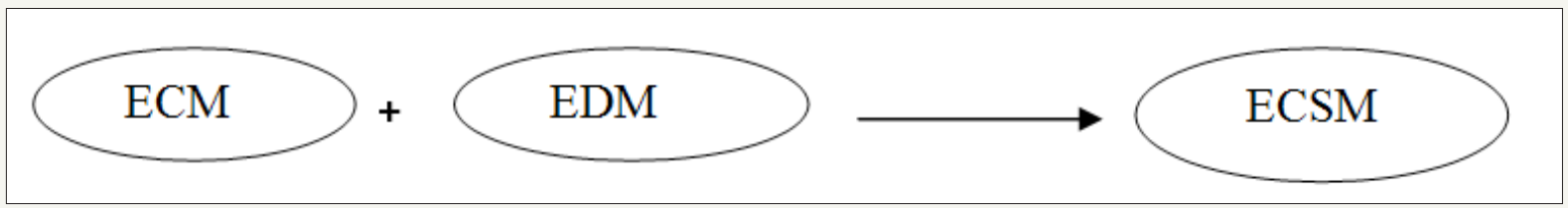

Figure 2

Electrochemical discharge machining (ECDM) is a hybrid machining process which combining the features of electrochemical machining (ECM) and electro discharge machining (EDM), however process is entirely different from ECM and EDM. The process is also known as electrochemical spark machining (ECSM) process. It was first presented in 1968 by Kurafuji \& Suda [2] as "Electrical discharge drilling for micro hole in glass". This hybrid machining process is successfully overcome the limitation of electrical conductivity of material. In ECDM material is removed by electrochemical discharge phenomena. The combined action of electro-chemical action and electro-spark action is called as electro-discharge action (Figure 2). In ECDM electrical discharge is generated inside a gas film surrounding the tool electrode for machining purpose [1].

In electrochemical discharge machining process, electrochemical discharge is observed between small electrode and electrolyte in a system of two electrode of different size, usually small cathode (in this case only one electrode use as tool, however auxiliary electrode can also be made small so that sparking zone may be available on both electrode [2], the different machining conditions are known as ECSMWDP and ECSMWRP), separated by a critical distance and when energised beyond a critical DC voltage. Electrochemical reaction at cathode and anode can be written as

Reaction at Cathode:

$$
\begin{aligned}
& 2 \mathrm{H}^{+}+2 e^{-} \rightarrow \mathrm{H}_{2} \uparrow \\
& 2 \mathrm{H}_{2} \mathrm{O}+2 e^{-} \rightarrow 2(\mathrm{OH})^{-}+\mathrm{H}_{2} \uparrow
\end{aligned}
$$

$$
\begin{aligned}
& \mathrm{Na}^{+}+e^{-} \rightarrow \mathrm{Na} \\
& 2 \mathrm{Na}+2 \mathrm{H}_{2} \mathrm{O} \rightarrow 2 \mathrm{NaOH}+\mathrm{H}_{2} \uparrow
\end{aligned}
$$

Reaction at anode:

$$
\begin{aligned}
& M \rightarrow M^{z+}+\mathrm{Ze}^{-} \\
& M^{z+}+\mathrm{Z}(\mathrm{OH})^{-} \rightarrow M(\mathrm{OH})_{z} \downarrow \\
& 2 \mathrm{H}_{2} \mathrm{O} \rightarrow \mathrm{O}_{2} \uparrow+4 H^{+}+4 e^{-}
\end{aligned}
$$

(In acidic electrolyte solution)

$$
4(\mathrm{OH})^{-} \rightarrow 2 \mathrm{H}_{2} \mathrm{O}+\mathrm{O}_{2} \uparrow+4 e^{-}
$$

(In alkaline electrolyte Solution)The above electro-chemical reactions are responsible for $\mathrm{H}_{2}$ and $\mathrm{O}_{2}$ gas bubble generation at cathode and anode respectively. These gas bubbles are detached from the tool electrode surface as long as they are smaller. Bubble density and its mean radius depends on the applied voltage, as applied voltage increases, bubble density and mean radius also increases. In this way at critical DC voltage, density of gas bubble production is so high that they coalesce into each other and strongly grip the tool electrode surface by capillary action. In this way a gas film is formed at tool electrode which isolates the tool electrode from electrolyte. The electrical field in this film is high enough (Typically $10^{6}-10^{8} \mathrm{~V} / \mathrm{m}$ ) to allow electric discharge between tool electrode and electrolyte [3]. This phenomenon is called as electrochemical discharge. Figure 3 shows electrochemical discharge phenomena. 


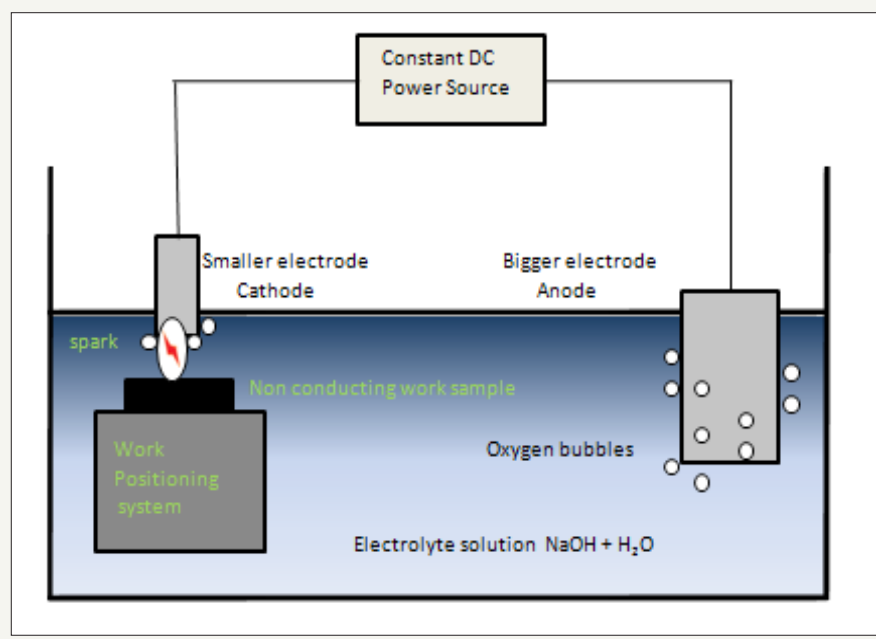

Figure 3: Schematic diagrams of electro-chemical discharge phenomena [1].

Machining with electrochemical discharge phenomena is possible only above the critical voltage. In this situation several processes may contribute to the machining mechanism. Following are some of them-

Melting and vaporization due to electrochemical discharges

1. High temperature dissolution

2. Differential expansion of constituents and weathering

3. Random thermal stresses and micro-cracking and spalling

4. Mechanical shock due to expanding gases and electrolyte movement

Besides all these potential mechanism until today only thermal effects and chemical etching were investigated.

Thermal machining is the most admitted hypothesis about the machining mechanism in ECSM phenomena. It is assumed that the work piece surface is intensively heated leading to melting and maybe even vaporization of the work material. Several analytical and FEM calculations support this mechanism. Allasu et al. [4] showed by a simple experiment that the heat produced during electrochemical discharge phenomenon is able to melt glass. Kulkarni et al. [5] showed by various measurements that after each discharge, the temperature of the work piece increases above the melting temperature and sometime even above the vaporization temperature of the machined material. They estimated that about $77 \%-96 \%$ of the energy supplied to the process is used to heat the electrolyte and only $2-6 \%$ is used for heating up the work piece. Another experimental evidence for thermal mechanism in machining is the various observations of thermal cracks insides the machined materials which are seen mainly at high voltage machining. The difficulty in this model is to estimate correctly the amount of energy per discharge.

Several observations indicate that not only thermal machining has some importance but some chemical etching too. Chemical etching also responsible for machining because the quality of machined surface depend strongly on the used electrolyte and it is also known that $\mathrm{NaOH}$ electrolyte leads to higher machining removal rate of glass than other ones. It is well known that $\mathrm{NaOH}$ etches glass by complexation of silicate.

$$
2 \mathrm{NaOH}+\mathrm{SiO}_{2} \rightarrow \mathrm{Na}_{2} \mathrm{SiO}_{3}(s)+\mathrm{H}_{2} \mathrm{O}
$$

This chemical reaction is strongly enhanced by increasing the temperature. Similar results are reported for ceramic materials.

\section{Literature Review}

The literature review of electro-chemical spark micromachining is summarized as:-Bhattacharyya et al. [6] studied the effect of applied voltage and electrolyte concentration on the process performance of sinking ECSMM during machining of aluminium oxide $\left(\mathrm{Al}_{2} \mathrm{O}_{3}\right)$ workpiece. They found that MRR increases with increases in applied voltage and electrolyte concentration, but overcut increases with increase in electrolyte concentration. They also observe that at higher voltage machined surface is affected by micro cracks and other defects. They concluded that the most effective range of applied voltage is centred on $80 \mathrm{~V}$ and concentration of $\mathrm{NaOH}$ electrolyte is around $25 \%$ leading the higher machining rate and dimensional accuracy. They also found that the tool tip shape is a prominent factor for controlling spark generation

Tandon et al. [7] carried out experiments to find the effects of change in applied voltage between electrodes and specific conductance of electrolyte on MRR, TWR and overcut during sinking ECSMM of Kevlar-fiber-epoxy and glass-fiber-epoxy composites. They found that, in case of glass-epoxy composite, MRR, TWR and overcut increase due to increase in applied voltage between electrodes and increase in specific conductance of electrolyte. In case of Kevlar-fiber-epoxy composite, MRR, TWR and radial overcut were increases with increase in specific conductance and decreases with tool diameter. Gautam \& Jain [8] conduct experiments using various tool kinematics and concluded that rotation of tool improves the process performance. However maximum machining depth is attained at low speed. Further increase in speed has an adverse effect on process performance. Limitation on the maximum depth has been partially overcome by the use of an orbital rotational tool. 
Laio et al. [9] studied the effect of sodium dodecyle surfactant added electrolyte on machining of quartz in ECSM and reported that the current density is increased, there is more bubble release around the electrode as compared with that when machining in electrolyte without sodium dodecyle sulphate. The spark become brighter and takes place in larger area, and a more stable pulse current is obtained. Chak \& Rao [10] uses the pulse DC power supply with three influential process parameter; applied voltage, duty factor and electrolyte conductivity to find their effect on MRR during machining of $\mathrm{SiC}$ with different electrode configuration. They reported that MRR increases with increase in supply voltage, duty factor, and electrolyte conductivity. Pulsating nature of power supply with proper duty factor restrict the flow of current and provide controlled heat at discharge zone and hence improved the machining performance and reduce the tendency of cracking at high supply voltage. They also reported that abrasive electrode with rotary motion provide additional electrical discharge which improve the machining rate.

Razfar \& Ni [11] studied the effect of cathode tool vibration in ECSM of glass. They reported that vibration have not significant effect on the process while a rotating micro drill is used as cathode electrode. But in case, when cylindrical rod is used as tool, vibration improves the MRR up to $40 \%$ and deeper hole can be achieved. Cheng et al. [12] studied the effect of applied voltage, and geometrical shape of tool on the gas film quality. They discussed the current signals and machined contours as index of gas film quality. They reported that, a stable and dense gas film could be obtained when applied voltage exceeded the critical voltage and reaches a specific level called as transition voltage. Change in shape of tool does not affect the structural stability of gas film, using a flat sidewall tool can mitigate the deterioration of gas film quality during drilling

\section{Different Configurations of ECSMM}

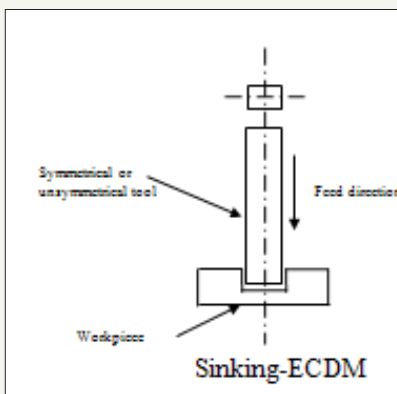

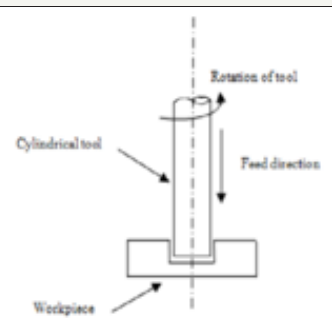

Drilling-ECDM process. Which in turn enhance machining efficiency. Efficiency of electrical discharge machining is depends on stability of gas film structure.

Wüthrich et al. [13] investigated that the factors affecting the machining speed at different machining depth are not same. At depth of 0-300 microns electrical discharge govern the machining speed, while depth exceeding 300 microns, circulation of electrolyte is dominating factor. Jain \& Adhikari [14] conducts experiment and concluded that; If both electrodes made small then machining can also take place at both the electrodes, it means both cathode and anode can be used as a tool. Now machining process is termed as ECSMWRP and ECSMWDP. ECMWRP cuts quartz plate at faster rate as compared to ECSMWDP, but produces higher overcut, higher tool wear and higher surface roughness

Zheng et al. [15] carried out experiments with applying a novel pulse voltage configuration called offset pulse voltage. The offset pulse voltage adds a constant voltage at $\mathrm{T}_{\text {off }}$ duration to enhance gas film stability in ECSM process improve the circulation of electrolyte and hence improves machining efficiency. Manna \& Narang [16] introduced an experimental investigation on the micro-machining of electrically non conducting e-glass-fiber-epoxy composite during ECSM using specially designed square cross section with centrally micro hole brass tool and different round shaped micro tool of IS-3748 steel. They developed a mathematical model for MRR and overcut and observed electrolyte concentration has the most significant effect on MRR and overcut. They found surface roughness during micro drilling of e-glass-fiber-epoxy composite is very poor and the reinforced epoxy fibers are not completely cut, rather they burnt and integrated. Jiang et al. [17] carried out experiments and concluded that Taper tool electrodes could enhance consistency of spark generation comparing with conventional tool.

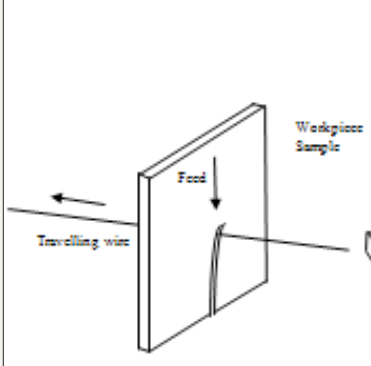

Travelling Wire-ECDM

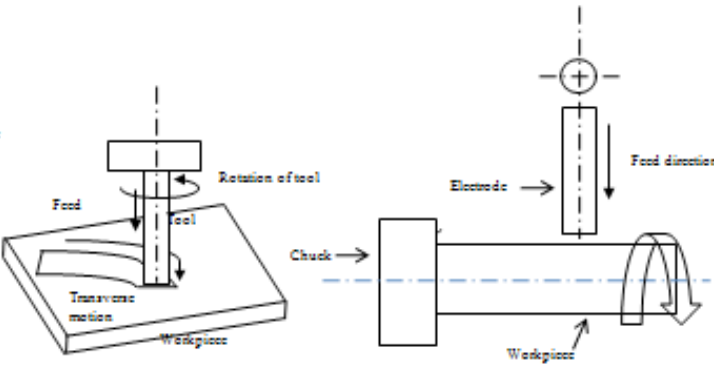

Milling-ECDM

Tuming-ECDM

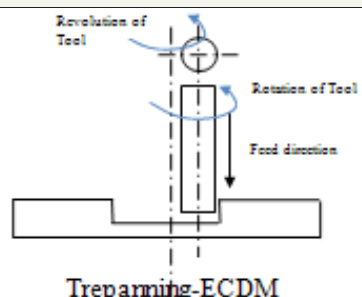

Treparning-ECDM

Figure 4: Different configurations of ECSMM. 
Electro-chemical discharges can be used to machine several electrically non-conductive materials. The machining mechanism is a combination of thermal and chemical machining; even this process known from 47 years, this machining process remains an academic application and was not applied in industrial production till now. The research done today mainly focused on experimenting the machining of various materials and investigating the effect of different parameters on the material removal rate. It was shown that a large class of materials (glass, quartz, various ceramics and others) can be machined. Not only simple structures as holes but as well as very complex structures like threads can be machined. Application of electro-chemical discharge has been found to machine micro features in four different ways such as Drilling ECSMM, Sinking ECSMM, Travelling wire ECSMM, and Milling ECSMM. The scheme of these configurations is shown in Figure 4 shows the different configuration possible for ECSMM process.

a. Sinking ECDM: ECSMM also used for hole sinking operations. In hole sinking operations non rotating tool is used. Drilling is used for making symmetrical circular hole whereas a sinking operation used for making any type of hole. Main components of sinking ECSMM are same as in Drilling ECSMM

b. Drilling ECDM: Hole drilling was the first application of electro-chemical discharge phenomena, which was proposed by Kurafuji \& Suda [2] in Japan in 1968. In drilling ECSMM a rotating tool electrode is used with the main focus on surface quality of side wall of hole. Following are the main components of drilling ECSMM setup Power supply system
A. Electrolyte supply system
B. Workpiece positioning system
C. Tool electrode feeding system
D. Vision system

The power supply system is mostly DC power supply. Pulsated DC may also apply to increase the performance of machining. Electrolyte supply system consists of electrolyte chamber, pump, and electrolyte tank. The machining chamber is mounted on a base by using some fixing arrangements. The chamber is well equipped for circulation of electrolyte flow system. There is a work piece holder in the chamber. All the parts including machining chamber and work piece holder should be made of corrosion resistant material. The tool electrode is needle shaped, made either by stainless steel or platinum or nickel. A constant force is applied on tool or work piece by gravity feed system.

c. Travelling wire ECSMM: Another use of ECSMM process is in machining of non conducting workpiece by using continues wire as an electrode. It is an extension to wire machining, called travelling wire electrochemical spark micro-machining (TWECSMM). Travelling wire ECSMM was first introduced by Tsuchiya et al. [18] and studied further on it by Jain et al. [19] and Peng et al. [20] TW-ECSMM is particularly interesting for slicing glass fiber composites, but may be used as well for 2D contours cutting. In this configuration a wire is used as tool in analogue way as in wire discharge machining (WEDM). Typical used wires are $0.9 \mathrm{~mm}$ cooper wire, $0.25 \mathrm{~mm}$ stainless steel wire, or 0.5 and $0.2 \mathrm{~mm}$ brass wire. The wire speed is a compromise between high speed in order to allow the cooling of the wire (avoiding overheating and breaking) and low speed for economical reasons. Typical speeds are a few mm per minute or a few $\mathrm{cm}$ per minute depending on the used set-up. The wire may be guided horizontally or vertically. Several materials like glass, quartz, alumina, PZT ceramics and various composites (glass and Kevlar-epoxy) can be machined with TWECSMM. The preferred electrolyte is in general $\mathrm{NaOH}$. The applied voltage may be D.C. voltage or pulsed one. Compared to hole drilling or $3 \mathrm{D}$ structuring the voltage is in general higher which is due to the different geometry of the wire (larger surface) compared to a cylindrical tool. Workpiece feeding is done by gravity or by constant speed (at very slow speed in order to be slower than the mean machining speed). The thickness of the work piece can be in the range of $1 \mathrm{~mm}^{-1} \mathrm{~cm}$ (for glass). The stabilization of the temperature during machining by appropriate flushing of the electrolyte is possible. Typically schematic diagram is shown in Figure 5.

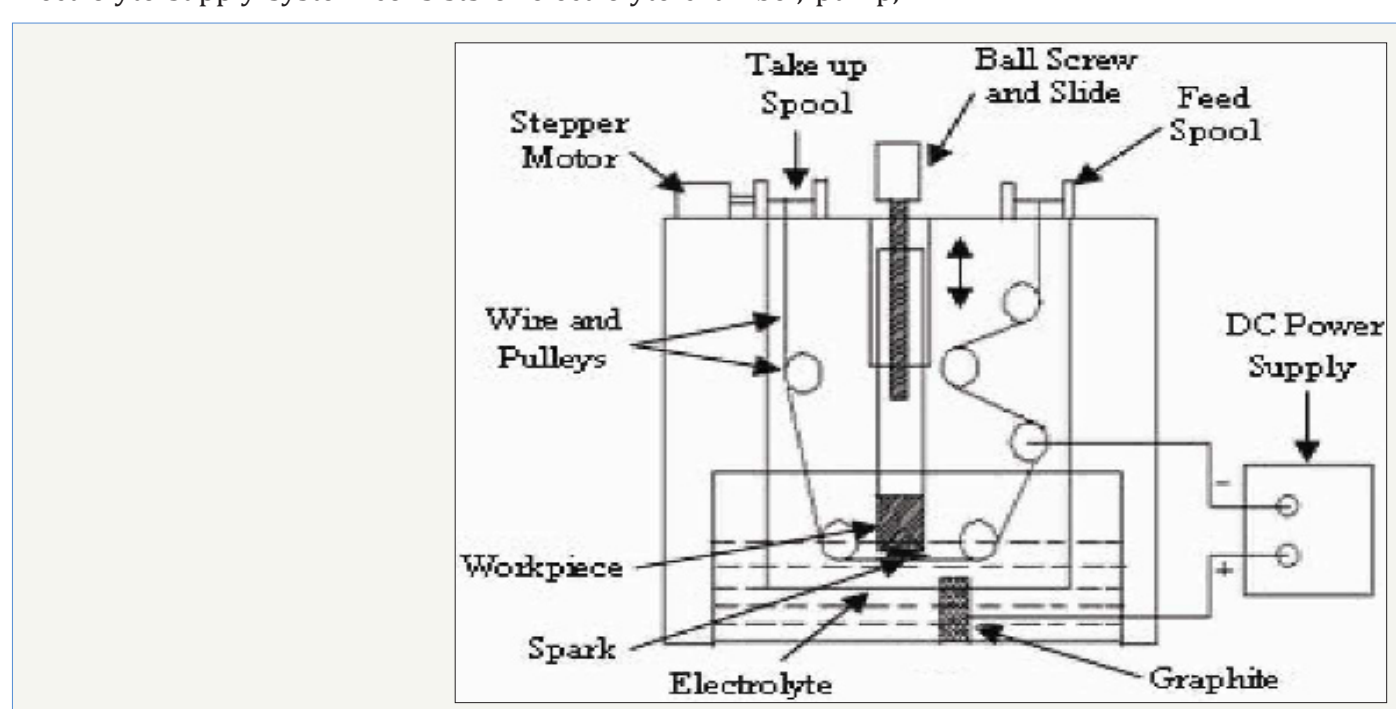

Figure 5: Schematic diagrams of TW-ECSM setup [21] 
The main components are following
A. Workpiece holder
B. Workpiece feeding system
C. Wire driving system
D. Power supply system
E. Machining chamber
F. Vision system

d. Milling ECSMM: A simple shaped micro sized electrode is used to produce three dimensional cavities by adapting a movement strategy similar to that in conventional milling. First Langen et al., have conducted experiments for 3D micro structuring of glass by milling-ECSMM. Trepanning-ECDM, Turning-ECDM and Grinding ECDM process need different setup acceding to their process parameters.

\section{Conclusion}

ECSMM process is suitable for advanced engineering materials, composites, ceramics, high performance steels, electrically nonconducting and conducting materials etc, with different available configurations provide ease of machining. Cutting of electrically non-conducting, hard and brittle material is always been a challenging task in manufacturing engineering. To perform through hole drilling using ECSMM process many challenges are risen, they are summarized as damage at the entrance and exit of hole, roughness of wall inside the hole, low machining rate, accuracy of hole, taper in hole. Much more research is required to overcome the above illustrated challenges. The commercialization of this process is only possible through the optimization of the process and achieving a satisfactory level of machining performance and dimensional accuracy.

\section{References}

1. Rajurkar KP, Tandon S, Jain VK (1990) Investigations into machining of composites. Precision Engineering 12(4): 227-238.

2. Kurafuji H, Suda K (1968) Electrical discharge drilling of glass, annals of the CIRP 16: 415-419.

3. Yerokhin, Nie X, Leyland A, Matthews A, Dowey SJ (1999) Plasma electrolysis for surface engineering. Surface and Coating Technology 122(2-3): 73-93

4. Allasu K, Manju MK, Ghosh A (1992) Preliminary qualitative approach of a proposed mechanism of material removal in electrical machining of glass. European journal of mechanical engineers 36: 202-207.

5. Kulkarni, Sharan R, Lal GK (2002) An experimental study of discharge mechanism in electrochemical discharge mechanism. International Journal of Machine Tools Manufacture 42: 1121-1127.
6. Bhattacharyya B, Doloi BN, Sorkhel SK (1999) Experimental investigation into electrochemical discharge machining of non-conductive ceramic materials. Journals of Materials Processing Technology 95(1-3): 145154.

7. Tandon S, Jain VK, Kumar P, Rajurkar KP (2008) Experimental investigations into electrochemical spark machining of composites. Journal of Engineering for Industry 17: 112.

8. Gautam N, Jain VK (1997) Experimental investigation into ECSD process using various tool kinematics. International Journal Machine Tool Manufacture 38(1-2): 15-27.

9. Laio YS, Wu LC, Peng WY (2013) A study to improve drilling quality of electrochemical discharge machining process. Procedia CIRP 6: 609 -614 .

10. Chak SK, Rao PV (2014) Machining of SiC by ECDM process using different electrode conguration under the effect pulsed DC. International Journal of Manufacturing Technology and Management 28(1-3).

11. Razfar MR, Ni J (2014) Study of the tool longitudinal oscillation on the machining speed of electrochemical discharge drilling of glass. Precision Engineering 38(4): 885-892.

12. Cheng CP, Wu KL, Mai CC, Yang CK, Hsu YS, et al. (2010) Study of gas film quality in electrochemical discharge machining. International Journal of Machine Tools and Manufacture 50(8): 689-697.

13. Wüthrich R, Fascio V (2005) Machining of non-conducting materials using electrochemical discharge phenomena-an overview. International Journal of Machine Tool and Manufacture 45(9): 1095-1108.

14. Jain VK, Adhikary S (2008) On the mechanism of material removal in electrochemical spark machining of quartz under different polarity conditions. Journal of materials processing technology 200(1-2): 460470 .

15.Zhang Y, Xu Z, Zhu D, Xing Z (2015) Tube electrode high speed electrochemical discharge drilling using low conductivity salt solutions. International Journal of Machine Tools and Manufacture 92: 10-18.

16. Manna A, Narang V (2012) A study on micro machining of e-glass fiber epoxy composite by ECSM process. International Journal of Advance Manufacturing Technology 61(9-12): 1191-1197.

17. Jiang B, Lan S, Ni J, Zhang Z (2014) Experimental investigation of spark generation in electrochemical discharge machining of non-conducting materials. Journal of material processing technology 214(4): 892-898.

18. Tsuchiya H, Inoue T, Miyazaiki M (1985) Wire electrochemical discharge machining of glass and ceramics bull. Japan Society of Precision Engineering 19: 73-74.

19. Jain VK, Dixit PM (1999) On the analysis of electrochemical spark machining process. International Journal of Machine Tools Manufacture 39(1): 165-186

20. Peng WY, Liao YS (2004) Study of electrochemical discharge technology for slicing non-conducting brittle material. Journal of Materials Processing Technology 149(1-3): 363-369.

21. Bhuyan BK, Vinod Y (2013) Experimental modelling and multi-objective optimization of travelling wire electrode chemical spark machining (TW-ECSM) process. JMST 27(8): 2467-2476. 
Creative Commons Attribution 4.0 International License

For possible submissions Click Here

Submit Article

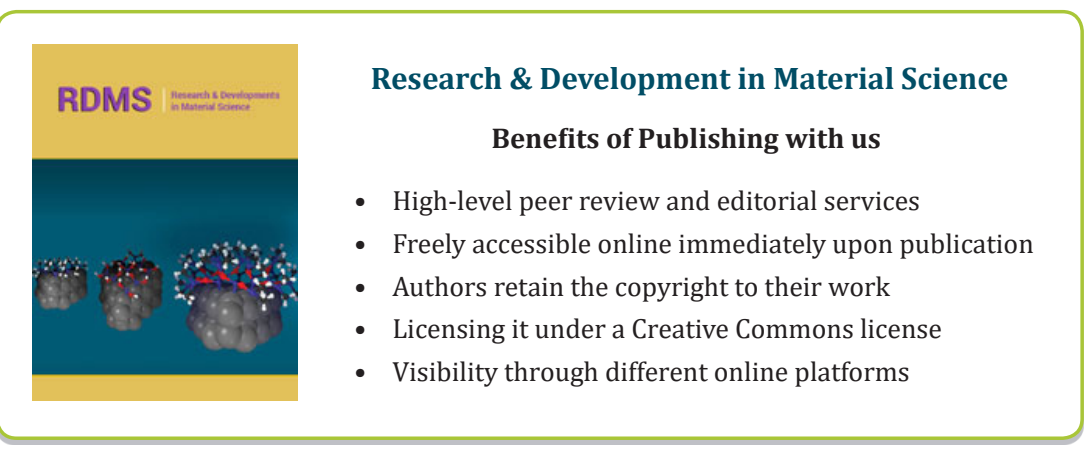

